\title{
Box Invariance for biologically-inspired dynamical systems
}

\author{
Alessandro Abate $\dagger \quad$ Ashish Tiwarił Shankar Sastry $\dagger$ \\ $\dagger$ Department of Electrical Engineering and Computer Sciences \\ University of California, at Berkeley - \{aabate, sastry\}@eecs .berkeley . edu \\ $\ddagger$ Computer Science Laboratory, SRI International \\ Menlo Park, CA - tiwariecsl.sri.com
}

\begin{abstract}
In this paper, motivated by models drawn from biology, we introduce the notion of box invariant dynamical systems. We argue that box invariance, that is, the existence of a "box"-shaped positively invariant region, is a characteristic of many biologically-inspired dynamical models. Box invariance is also useful for the verification of stability and safety properties of such systems. This paper presents effective characterization of this notion for some classes of systems, computational results on checking box invariance, the study of the dynamical properties it subsumes, and a comparison with related concepts in the literature. The concept is illustrated using models derived from different case studies in biology.
\end{abstract}

\section{INTRODUCTION}

A positively invariant set is a subset of the state space of a dynamical system with the property that, if the system state is in this set at some time, then it will continue to be in this set in the future [1]. A positively invariant set is extremely useful from the perspective of formal analysis and verification. It can be used to verify safety properties of a system, that is, properties that specify that a system can never be in a prespecified subset of "unsafe" or "bad" states, as well as stability specifications [2]. This motivates the need for an effective constructive approach to compute positively invariant sets for dynamical systems.

Positively invariant sets can be obtained by noticing that the level surfaces of a Lyapunov function form boundaries of positively invariant sets. This approach has been a source of several results about positively invariant sets. However, this is quite restrictive in general, since systems that are not stable (and hence that do not have Lyapunov functions) can also have useful invariant sets.

In this paper, we focus on positively invariant sets that are in the form of a box, that is, a region specified by giving bounds for each state variable. The investigation of several models, especially from the domain of Systems Biology, has revealed that they frequently admit box-shaped positively invariant sets. This seems natural in retrospect since state variables often correspond to physical quantities that are naturally bounded. We show in this paper that it is computationally feasible to construct box invariant sets for a large class of dynamical systems and hence it is an ideal concept for building analysis and verification tools.

In this manuscript we introduce and define the notion of box invariance. We start with the simplest instances of linear

Research supported by the grants CCR-0225610 and DAAD19-03-10373, and in part by NSF CCR-0311348 and DARPA DE-AC03-765F00098. and affine dynamical systems and move to more general nonlinear systems. We present computational complexity results on computing box invariant sets. The proofs of the claims, all originally derived, can be found in [3]. Box invariance of linear systems is strongly related to the theory of Metzler matrices and we use this connection to study the dynamical properties and perform robustness analysis of box invariant systems. We present several examples from Systems Biology to demonstrate the significance of the concept of box invariance and also present a comparison with other strongly related concepts in the systems and control theory literature.

\section{THE CONCEPT OF BOX INVARIANCE}

In this work, we shall consider general, autonomous and uncontrolled dynamical systems of the form $\dot{\boldsymbol{x}}=f(\boldsymbol{x}), \boldsymbol{x} \in$ $\mathbb{R}^{n}$. We assume the basic continuity and Lipschitz properties for the existence of a unique solution of the vector field, given any possible initial condition. A rectangular box around a point $\boldsymbol{x}_{0}$ can be specified using two diagonally opposite points $\boldsymbol{x}_{l b}$ and $\boldsymbol{x}_{u b}$, where $\boldsymbol{x}_{l b}<\boldsymbol{x}_{0}<\boldsymbol{x}_{u b}$ (interpreted component-wise). Such a box has $2 n$ surfaces $S^{j, k}(1 \leq j \leq n, k \in\{l, u\})$, where $S^{j, k}=\left\{\boldsymbol{y}: x_{l b, i} \leq y_{i} \leq\right.$ $x_{u b, i}$ for $i \neq j, y_{j}=x_{l b, j}$ if $k=l, y_{j}=x_{u b, j}$ if $\left.k=u\right\}$.

Definition 1: A dynamical system $\dot{\boldsymbol{x}}=f(\boldsymbol{x})$ is said to be box invariant around an equilibrium point $\boldsymbol{x}_{0}$ if there exists a finite rectangular box around $\boldsymbol{x}_{0}$, specified by $\boldsymbol{x}_{l b}$ and $\boldsymbol{x}_{u b}$, such that for any point $\boldsymbol{y}$ on any surface $S^{j, k}(1 \leq j \leq n, k \in$ $\{l, u\})$ of this rectangular box, it is the case that $f(\boldsymbol{y})_{j} \leq 0$ if $k=u$ and $f(\boldsymbol{y})_{j} \geq 0$ if $k=l$. The system will be said to be strictly box invariant if the last inequalities hold strictly.

Remark 1: An equivalent definition of box invariant systems can be given as systems that have a box as a positively invariant set. The equivalence of these two definitions follows from a basic result concerning positive invariance that states that a closed and convex set $B$ is positively invariant for a system $\dot{\boldsymbol{x}}=f(\boldsymbol{x})$ iff for all points $\boldsymbol{x}$ on the boundary of $B$, $f(\boldsymbol{x})$ is in the tangent cone of $B$ at $\boldsymbol{x}$, see [1].

Remark 2: The concept of box invariance requires the existence of a positively invariant set for a dynamical system with a special polyhedral shape. In the case of linear systems, this invariant set is also a domain of attraction and its existence will imply stability (towards the enclosd equilibrium): hence, a notion of box stability (see Theorem 5).

Note that the existence of a box is unaffected by the reordering of state variables and the rotations by multiples of 
$\pi / 2$; it also displays invariance under independent stretches of the coordinates. Nevertheless, it is not invariant under general linear transformations.

Definition 2: A system $\dot{\boldsymbol{x}}=f(\boldsymbol{x})$ is said to be symmetrical box invariant around the equilibrium $\boldsymbol{x}_{0}$ if there exists a point $\boldsymbol{u}>\boldsymbol{x}_{0}$ (interpreted component-wise) such that the system $\dot{\boldsymbol{x}}=f(\boldsymbol{x})$ is box invariant with respect to the box defined by $\boldsymbol{u}$ and $\left(2 \boldsymbol{x}_{0}-\boldsymbol{u}\right)$.

\section{A. Vector Norms}

The boundary of a box can be seen as a level surface of a vector norm. Let $\|\boldsymbol{x}\|_{\infty}=\max \left\{\left|x_{i}\right|, i=1, \ldots, n\right\}$ denote the infinity norm. Let $D$ be a $n \times n$ positive diagonal matrix. The level set of $\|D \boldsymbol{x}\|_{\infty}$ is a hyper-rectangle in $\mathbb{R}^{n}$ that is symmetric around the origin.

Symmetrical box invariance has been studied in the literature by exploring when $\|D \boldsymbol{x}\|_{\infty}$ is a Lyapunov function for a dynamical system. In fact, the notion of componentwise (exponential) asymptotic stability of a linear system is exactly characterized by $\|D \boldsymbol{x}\|_{\infty}$ being a strong Lyapunov function [4].

More generally, for a linear system $\dot{\boldsymbol{x}}=A \boldsymbol{x}$, a vector norm $\|W \boldsymbol{x}\|$, where $W$ is of rank $n$, will be a Lyapunov function if $\mu(Q)<0$, where $W A=Q W$ [5]. This condition is also sufficient for quadratic and infinity norms [6]. Here $\mu(Q)$ is a matrix measure defined by $\lim _{\Delta \rightarrow 0^{+}} \frac{\|I+\Delta Q\|-1}{\Delta}$.

In this paper, we focus on box invariance. Though this is closely tied to the notion of asymptotic stability for linear systems, these two concepts are incomparable in general. The emphasis here is on the computational results about box invariance for linear and nonlinear systems and a study of robustness.

\section{Characterization of Box Invariance.}

We investigate the notion of box invariance for several classes of systems, propose efficient computational ways to find such boxes, and study their robustness properties.

\section{A. Linear Systems.}

Given a linear system and a box around its equilibrium point, the problem of checking if the system is box invariant with respect to the given box can be solved by checking the condition only at the $2^{n}$ vertices of the box (instead of on all points of all the faces of the box).

Proposition 1: A linear system $\dot{\boldsymbol{x}}=A \boldsymbol{x}, \boldsymbol{x} \in \mathbb{R}^{n}$ is box invariant if there exist two points $\boldsymbol{u} \in \mathbb{R}^{+n}$ and $\boldsymbol{l} \in \mathbb{R}^{-n}$ such that for each point $\boldsymbol{c}$, with $c_{i} \in\left\{u_{i}, l_{i}\right\}, \forall i$, we have $A c \sim \mathbf{0}$, where $\sim_{i}$ is $\leq$ if $c_{i}=u_{i}$ and $\sim_{i}$ is $\geq$ if $c_{i}=l_{i}$.

Remark 3: Proposition 1, which is a simple consequence of linearity, shows that box invariance of linear systems can be checked by testing satisfiability of $n 2^{n}$ linear inequality constraints (over $2 n$ unknowns given by $\boldsymbol{l}$ and $\boldsymbol{u}$ ). Theorem 1 and Theorem 2 will allow us to simplify this to testing $n$ linear inequalities over $n$ variables.

The notion of box invariance and symmetrical box invariance are equivalent for linear systems:

Theorem 1: A linear system $\dot{\boldsymbol{x}}=A \boldsymbol{x}$, where $A \in \mathbb{R}^{n \times n}$, is box invariant iff it is symmetrical box invariant.
As a result of Theorem 1, we can now use results obtained using infinity vector norms as Lyapunov functions [5], [4]. The following result can be easily obtained using a direct proof based on simplifying the $n 2^{n}$ inequality constraints.

Theorem 2: An $n$-dimensional linear system $\dot{\boldsymbol{x}}=A \boldsymbol{x}$ is symmetrical box invariant iff there exists a positive vector $\boldsymbol{c} \in \mathbb{R}^{+n}$ such that $A^{m} \boldsymbol{c} \leq \mathbf{0}$, where $a_{i i}^{m}=a_{i i}(<0)$ and $a_{i j}^{m}=\left|a_{i j}\right|$ for $i \neq j$. This is equivalent to checking if the linear system defined by $A^{m}$ is symmetrical box invariant.

Putting together Theorem 1 and 2, we conclude that in order to check whether a linear system $\dot{\boldsymbol{x}}=A \boldsymbol{x}$ is box invariant, we only need to test if there exists a positive vector $c$ such that $A^{m} c \leq 0$. This can be solved using linear programming in polynomial time. However, we can do much better. Since $A^{m}$ has negative diagonal terms and non-negative off-diagonal terms, it is immediate that Fourier-Motzkin procedure can be used to solve the $n$ linear inequality constraints $A^{m} \boldsymbol{c} \leq 0$ for positive $\boldsymbol{c}$ in $O\left(n^{3}\right)$ time.

In fact, we can exactly characterize when the FourierMotzkin procedure would succeed in finding a solution using the notion of principal minors. A principal minor of a matrix $A$ is the determinant of the submatrix of $A$ formed by removing certain rows and the same columns from $A$, [7]. In particular, the diagonal elements of $A$ are its principal minors and the determinant of $A$ is also a principal minor. A matrix $A$ is said to be a $P$-matrix if all of its principal minors are positive.

Theorem 3: Let $A$ be a $n \times n$ matrix such that $a_{i i} \leq 0$ and $a_{i j} \geq 0$ for all $i \neq j$. Then, the following statements are equivalent:

1) The linear system $\dot{\boldsymbol{x}}=A \boldsymbol{x}$ is strictly symmetrical box invariant.

2) $-A$ is a $P$-matrix.

3) For every $i=1,2, \ldots, n$, the determinant of the top left $i \times i$ submatrix of $-A$ is positive.

Remark 4: Theorem 3 shows that box invariance of linear systems can also be tested by checking if the modified matrix $-A^{m}$ is a $P$-matrix. It is known that the problem of deciding if a given matrix is a $P$-matrix is co-NP-hard (see [8], [9]). But in our case, due to the special form of $A^{m}$, we can determine if $-A^{m}$ is a $P$-matrix using a simple $O\left(n^{3}\right)$ Fourier-Motzkin elimination procedure.

In the language of infinite vector norms, the existence of a positive vector $\boldsymbol{c}$ such that $A^{m} \boldsymbol{c} \leq 0$ is equivalent to $\mu\left(D^{-1} A^{m} D\right) \leq 0$, where $D$ is the positive diagonal matrix $\operatorname{diag}(\boldsymbol{c})$. This connection was known [5], [4], but we now have the following new complexity result.

Theorem 4: Let $A \in \mathbb{Q}^{n \times n}$ be any matrix and let $A^{m}$ denote any rational matrix such that $a_{i i}^{m}<0$ and $a_{i j}^{m} \geq 0$ for $i \neq j$ (e.g., the one obtained from $A$ ). The following problems can be solved in $O\left(n^{3}\right)$ time:

- Is the linear system $\dot{\boldsymbol{x}}=A \boldsymbol{x}$ (strictly) box invariant?

- Are the constraints $A^{m} \boldsymbol{z} \leq 0, \boldsymbol{z}>0$ satisfiable?

- Does there exist a positive diagonal matrix $D$ s.t. $\mu\left(D^{-1} A^{m} D\right) \leq 0$ (in the infinity norm)?

- Is $-A^{m}$ a $P$-matrix?

Remark 5: Theorem 4 is stated for rational matrices since 
irrational real numbers are computationally difficult to represent and manipulate.

We can not only decide box invariance, but also find box invariant sets by generating solutions for the above linear constraint satisfaction problem. Indeed, with a linear system, $\dot{\mathbf{x}}=A \mathbf{x}$, we can associate a cone in the positive $2^{n^{t h}}$-ant described by the set

$$
\mathcal{C}=\left\{\boldsymbol{x} \in \mathbb{R}^{+^{n}}: A^{m} \boldsymbol{x} \leq \mathbf{0}\right\} .
$$

Any choice of a single vertex in $\mathcal{C}$, or a couple of different points in $\mathrm{C}$ and its origin-symmetric, determine respectively a symmetric and a non-symmetric box for the system described by $A$ (see Fig. 1). degree at most one in each monomial. Multi-affine systems have several nice properties that have been exploited for building efficient analysis tools. We generalize the definition of multi-affine systems and call a system $\dot{\boldsymbol{x}}=\boldsymbol{p}(\boldsymbol{x})$ multiaffine if each variable $x_{j}$ has degree at most one in each monomial in $p_{i}$ for all $j \neq i$. In fact, the universal quantifiers in Formula (1) can be eliminated and Formula (1) can be simplified for multi-affine systems to a conjunction of (existentially quantified) $n 2^{n}$ constraints using the following analogue of Proposition 1 .

Proposition 2: A multi-affine system $\dot{\boldsymbol{x}}=\boldsymbol{p}(\boldsymbol{x}), \boldsymbol{x} \in \mathbb{R}^{n}$ is box invariant iff there exist two points $\boldsymbol{l}, \boldsymbol{u} \in \mathbb{R}^{n}$ such that for each point $\boldsymbol{c}$, with $c_{i} \in\left\{u_{i}, l_{i}\right\}, \forall i$, we have $\boldsymbol{p}(\boldsymbol{c}) \sim \mathbf{0}$, where $\sim_{i}$ is $\leq$ if $c_{i}=u_{i}$ and $\sim_{i}$ is $\geq$ if $c_{i}=l_{i}$.

Proposition 2 still requires checking satisfiability of an exponential number of (nonlinear) constraints. The following result shows that we can not hope to obtain polynomial time algorithms for checking box invariance of multi-affine systems for the case when the box is given.

Theorem 7: The problem of determining if a multi-affine system is box invariant with respect to a given box is coNP-hard.

Proof: (Sketch) Given a clause $\phi$, say $b_{1} \vee \overline{b_{2}} \vee \overline{b_{3}}$, let $\operatorname{poly}(\phi)$ denote the polynomial $\left(1-x_{1}\right) x_{2} x_{3}$. Given a formula $\phi$ consisting of the clauses $\phi_{i}$, let $\operatorname{poly}(\phi)$ denote the polynomial $\Sigma_{i}$ poly $\left(\phi_{i}\right)$. Suppose $\phi$ is an instance of 3SAT with $n$ boolean variables. Consider the following multiaffine system (note that this system is multi-affine even in

the sense of [12]),

Fig. 1. A three-dimensional conic region $\mathcal{C}$ describing all possible choices for the positive vertex of an jnvariant box.

For linear systems, box invariance is a stronger concept than stability, see also [5], [4], [6].

Theorem 5: If a linear dynamical system is box invariant, then it is stable.

\section{B. Nonlinear Systems.}

Dynamical models in biology are often in the form of polynomial systems, $\dot{\boldsymbol{x}}=\boldsymbol{p}(\boldsymbol{x})$, where $\boldsymbol{p}(\boldsymbol{x})$ is a vector of polynomials over $\boldsymbol{x}$. The condition for box invariance of polynomial systems can be written as a formula in the first-order theory of reals

$$
\begin{array}{r}
\exists \boldsymbol{l}, \boldsymbol{u} . \forall \boldsymbol{x} . \quad \bigwedge_{1 \leq j \leq n} \quad\left(\left(\boldsymbol{x} \in S^{j, l} \Rightarrow \boldsymbol{p}_{j}(\boldsymbol{x}) \geq 0\right) \wedge\right. \\
\left.\left(\boldsymbol{x} \in S^{j, u} \Rightarrow \boldsymbol{p}_{j}(\boldsymbol{x}) \leq 0\right)\right),
\end{array}
$$

where, as mentioned earlier, $S^{j, k}$ are the $2 n$ faces of the box defined by $\boldsymbol{l}$ and $\boldsymbol{u}$. Since this theory is decidable [10], [11], the following result follows.

Theorem 6: Box invariance of polynomial systems is decidable.

While this is a useful theoretical result, it is not very practical due to the high complexity of the decision procedure for real-closed fields. A subclass of polynomial systems, called multi-affine systems [12], arise naturally in modeling biochemical reaction networks [12], [13]. In these systems, the polynomials are restricted so that each variable has $\dot{x}_{i}=-x_{i},(i=1, \ldots, n) ; \dot{x}_{n+1}=x_{n+1}(1-\operatorname{poly}(\phi))$,

and the box given by $\mathbf{0} \leq \boldsymbol{x} \leq 1$. It can be shown that this box is positively invariant for the multi-affine system iff $\phi$ is unsatisfiable.

However, for a very useful subclass of multi-affine systems, we can reduce the number of constraints (from $n 2^{n}$ ) to $2 n$. We use the notion of monotonicity-function $f$ : $\mathbb{R}^{n} \mapsto \mathbb{R}$ is monotonic with respect to a variable $x_{j}$ if $f\left(\ldots, x_{j}, \ldots\right) \leq f\left(\ldots, x_{j}^{\prime}, \ldots\right)$ (or $f\left(\ldots, x_{j}, \ldots\right) \geq$ $\left.f\left(\ldots, x_{j}^{\prime}, \ldots\right)\right)$ whenever $x_{j}<x_{j}^{\prime}$.

Proposition 3: Let $\dot{\boldsymbol{x}}=\boldsymbol{p}(\boldsymbol{x})$ be a multi-affine system such that each multi-affine polynomial $\boldsymbol{p}_{i}(\boldsymbol{x})$ is monotonic with respect to every variable $x_{j}$ for $j \neq i$. Then, the $n 2^{n}$ constraints of Proposition 2 are equivalent to some subset of $2 n$ constraints.

We illustrate the ideas and the utility of Proposition 3 in the following example.

Example 1: Consider the following Phytoplankton Growth Model (see [14] and references therein):

$\dot{x}_{1}=1-x_{1}-\frac{x_{1} x_{2}}{4}, \quad \dot{x}_{2}=\left(2 x_{3}-1\right) x_{2}, \quad \dot{x}_{3}=\frac{x_{1}}{4}-2 x_{3}^{2}$,

where $x_{1}$ denotes the substrate, $x_{2}$ the phytoplankton biomass, and $x_{3}$ the intracellular nutrient per biomass. This system is not multi-affine in the sense of [12], but it is multi-affine in our weaker sense. Moreover, it satisfies the monotonicity condition, and hence by Proposition 3 , its box invariance is equivalent to the existence of $\boldsymbol{l}, \boldsymbol{u}$ s.t. 
the following 6 constraints (that subsume the $3 \cdot 2^{3}=24$ constraints) are satisfied:

$$
\begin{aligned}
& 1-u_{1}-\frac{u_{1} l_{2}}{4} \leq 0, \quad u_{2}\left(2 u_{3}-1\right) \leq 0, \quad \frac{u_{1}}{4}-2 u_{3}^{2} \leq 0, \\
& 1-l_{1}-\frac{l_{1} u_{2}}{4} \geq 0, \quad l_{2}\left(2 l_{3}-1\right) \geq 0, \quad \frac{l_{1}}{4}-2 l_{3}^{2} \geq 0 .
\end{aligned}
$$

One possible solution for these constraints is given by $\boldsymbol{l}=$ $(0,0,0)$ and $\boldsymbol{u}=(2,1,1 / 2)$ indicating that the box formed by these two points as diagonally opposite vertices is a positive invariant set.

The results on checking box invariance of multi-affine systems also generalize to a special class of hybrid systems wherein the system is described by a partition of the state space into finitely many subspaces and the system has multiaffine dynamics in each subspace partition. In other words, these are hybrid systems with finitely many discrete modes such that the state invariants of the modes partition $\mathbb{R}^{n}$ (thus the state uniquely determines the mode) and all reset maps (for all discrete transitions) are identity. These systems occur frequently as models of genetic regulatory pathways. More formal extensions of the study to hybrid and switched models has been discussed in [15]. The following example provides an illustrative example.

Example 2: The dynamics of tetracycline antibiotic in a bacteria which develops resistance to this drug (by turning on genes TetA and TetR) can be described by the following hybrid system, where $x_{1}, x_{2}, x_{3}, x_{4}$ are the cytoplasmic concentrations of TetR protein, the TetR-Tc complex, Tetracycline, and TetA protein, and $u_{0}$ is the extracellular concentration of Tetracycline [16]:

$$
\begin{array}{ll}
\dot{x}_{1}=f-\frac{x_{3} x_{1}}{3}+\frac{5 x_{2}}{40000} & \dot{x}_{2}=\frac{15 u_{0}}{1000}-\frac{35 x_{3} x_{4}}{10} \\
\dot{x}_{3}=\frac{x_{3} x_{1}}{3}-\frac{16 x_{2}}{40000} & \dot{x}_{4}=f-\frac{11 x_{4}}{40000}
\end{array}
$$

and $f=1 / 2000$ if Tet $>2 / 100000$ and $f=1 / 40$ otherwise ( $f$ is the transcription rate of genes, which are inhibited by TetR). In the mode when the genes are "on" (i.e., $f=1 / 2000$ ), if $u_{0}$ is fixed to 200 , then we can compute a positive invariant box $3 / 2 \leq x_{4} \leq 2,2 / 5 \leq$ $x_{3} 3 / 5,3 / 1000 \leq x_{1} \leq 8 / 1000,1 \leq x_{2} \leq 4$ essentially by focusing on the mode when the genes are "on" and using Proposition 3.

\section{Metzler Matrices.}

Matrices with the shape of those in Theorem 3 (or, equivalently, of $A^{m}$ in Theorem 2) are known under the appellative of Metzler matrices ${ }^{1}$. Metzler matrices are in fact, by definition, matrices with non-negative off-diagonal terms. In particular, the known positive matrices form a subset of them. Stochastic matrices (or rates matrices, which can be obtained from probability transition matrices) are another instance of Metzler matrices, with an additional constraint on the row sum; similarly for doubly stochastic matrices.

The properties of Metzler matrices can be reconducted to those of positive matrices, or at least to those of non-negative matrices; in fact, for every $A^{m} \in \mathbb{R}^{n \times n}$ that is Metzler, there exists a positive number $c$ such that $A^{m}+c I$ is non-negative. For instance, pick $c \geq \max _{i \in\{1, \ldots, n\}}\left|a_{i i}\right|$.

\footnotetext{
${ }^{1}$ Metzler matrices are also known as essentially non-negative matrices.
}

Perron and Frobenius (see refs. [17], [18]) were the first to study positive matrices; many results can be extended to the Metzler case provided a structural property, that of irreducibility, holds ${ }^{2}$. This property is also used in the theory of Markov Chains, and assumes that there's a connectivity chain between each pair of elements of the matrix, i.e. a sequence of links that brings from the first term of the couple to the second one, along the underlying connection graph that is associated with the matrix. In practical instances, this assumption is not restrictive, as its lack of validity would imply a certain level of decoupling between parts of the dynamical system; this would then advocate a separate study of these different parts in the first place, therefore solving the issue at its root. Example 4 will show this explicitly. Similar, slightly slacker results, can in any case be derived for the general case.

The following holds, (cf. [19]):

Proposition 4: Suppose $A^{m} \in \mathbb{R}^{n \times n}$ is Metzler; then it has an eigenvalue $\tau$ which verifies the following statements:

1) $\tau$ is real;

2) $\tau>\operatorname{Re}(\lambda)$, where $\lambda$ is any other eigenvalue of $A^{m}$ different from $\tau$;

3) $\tau$ has single algebraic and geometric multiplicity;

4) $\tau$ is associated with a unique (up to multiplicative constant) positive (right) eigenvector (equivalently, considering the transpose of $A^{m}$, also with a positive left eigenvector);

5) $\tau \leq 0$ iff $\exists \boldsymbol{c}>\mathbf{0}$, such that $A^{m} \boldsymbol{c} \leq \mathbf{0} ; \tau<0$ iff there is at least one strict inequality in $A^{m} \boldsymbol{c} \leq \mathbf{0}$;

6) $\tau<0$ iff all the principal minors of $-A^{m}$ are positive; 7) $\tau<0$ iff $-\left(A^{m}\right)^{-1}>0$.

Such a special $\tau$ is generally known as the PerronFrobenius eigenvalue of the matrix. We can prove the following theorem:

Theorem 8: Suppose $A^{m}$ is Metzler and has negative diagonal terms; then all the points of the previous fact hold but 5), which needs to be modified as:

5) $\tau \leq 0$ iff $\exists \boldsymbol{c}>\mathbf{0}$, such that $A^{m} \boldsymbol{c} \leq \mathbf{0} ; \tau<0$ iff $\exists \boldsymbol{c}>\mathbf{0}$, such that $A^{m} \boldsymbol{c}<\mathbf{0}$.

The following are two interesting results which will be useful in the sequel:

Theorem 9: If $A$ and $B$ are two Metzler matrices and $a_{i j, i \neq j} \leq b_{i j, i \neq j}$, while $a_{i i}=b_{i i}, \forall i \in\{1, \ldots, n\}$; then $\tau_{A} \leq \tau_{B}$, where $\tau_{A}, \tau_{B}$ are the two Perron-Frobenius eigenvalues of, respectively, $A$ and $B$.

Theorem 10: Given a Metzler matrix $A^{m}$, with PerronFrobenius eigenvalue $\tau$, the following holds:

$$
\min _{i} \sum_{j=1}^{n} a_{i j}^{m} \leq \tau \leq \max _{i} \sum_{j=1}^{n} a_{i j}^{m}, \quad i \in\{1, \ldots, n\} .
$$

If the equality holds, then it happens so in both directions.

Remark 6: A similar result holds calculating along the columns of the matrix $A^{m}$.

\footnotetext{
${ }^{2}$ For the sake of completeness, it has to be reported that another stronger assumption, that of primitivity, is also introduced to strengthen the results obtained for the irreducibility case.
} 
The previous results are interesting because they allow us to reinterpret the conditions we found beforehand (Thm. 2) within a new perspective. In particular, this sheds some light on Theorem 3, and proves it. If our original state matrix $A^{m}$ is already Metzler, then we can infer some dynamical properties of the linear system associated to it. For instance,

Corollary 1: Strict box invariance for a linear system $\dot{\boldsymbol{x}}=$ $A^{m} \boldsymbol{x}$, with $A^{m}$ Metzler, implies asymptotic stability. The converse is not true.

The following result, anticipated in the introduction, is interesting from a robustness study perspective.

Corollary 2: Given a Metzler matrix $A^{m}$, its box invariance is not affected by pre- or post-multiplications by positive diagonal matrices.

Although the connection with the theory of Metzler matrices appears quite promising, the reader should notice that in general it is not possible to directly translate results obtained for a Metzler matrix $A^{m}$ to its ancestor $A$, which may not be Metzler. In fact, the system associated with matrix $A^{m}$ is not always an upper bound for the original system associated with $A$; in particular, this setback happens for some combinations of the signs of the elements of the matrix, and, correspondingly, in some of the quadrants of the state space. Of course, if we pose restrictions on the signs of the variables (which may derive from the particular instances we may be considering), we may be allowed to exploit these bounds. The results outlined for the Metzler correspondent of a system matrix can be instead fully exploited for robustness analysis, as in the next section.

\section{Robust Properties of Box Invariance - Linear Systems}

The issue of robustness arises in biological models when some parameters of the system are not known exactly and thus may be thought to vary within specified bounds. These parameters may represent rates of reactions that are often unknown or subject to noise.

The theory of Metzler matrices allows us to exploit some results on the spectral properties of this class of matrices to study robustness of box invariance of linear systems. Consider Theorems 9 and 10. As discussed above, the positive Perron eigenvector $\boldsymbol{x}^{\tau}$ defines (one vertex of) the actual box; this knowledge can be exploited for obtaining stricter bounds for the Perron eigenvalue $\tau$.

Corollary 3: Given a Metzler matrix $A^{m}$, with PerronFrobenius eigenvalue $\tau$ and a positive vector $\boldsymbol{x}$, the following holds:

$\min _{i} \frac{1}{x_{i}} \sum_{j=1}^{n} x_{j} a_{i j}^{m} \leq \tau \leq \max _{i} \frac{1}{x_{i}} \sum_{j=1}^{n} x_{j} a_{i j}^{m}, i \in\{1, \ldots, n\}$.

Remark 7: The substitution of $\boldsymbol{x}^{\tau}$ in place of $\boldsymbol{x}$ turns the inequality into equality, in both directions. Thus, due to the continuous dependence of the eigenvalues of a matrix on its elements, the use of $\boldsymbol{x}=\boldsymbol{x}^{\tau}$ for bounding the value of $\tau$ of a matrix $A^{m}$ will definitely yield better results than the use of $\boldsymbol{x}=\mathbf{1}$, as in Thm. 10 .

If the Perron-Frobenius eigenvector is unknown, we can obtain improved bounds for the Perron-Frobenius eigenvalue independently of the computation of any vector. We skip the details, but refer the reader to the results for positive matrices in [20] for simple adaptation to the Metzler case.

We start with two cases in our study of robustness. The first deals with uncertainty on the diagonal terms, while the second with uncertainty on the off diagonal terms. It is clear that, for a matrix with Metzler form, the effect of these two sets towards box invariance is dichotomic: while the first contributes to it, the second can be disruptive.

1) Diagonal Perturbations: For the first instance, let us refer to a matrix of the form $A_{\epsilon}^{m}$, where $a_{\epsilon, i j}^{m}=a_{i j}^{m}, i \neq$ $j$, while $a_{\epsilon, i i}^{m}=a_{i i}^{m}(1+\epsilon)$; in other words, $A_{\epsilon}^{m}=A^{m}+$ $\epsilon \operatorname{diag}\left(a_{i i}^{m}\right)$. If $\epsilon>0$, then the perturbed system remains box invariant. If $\epsilon<0$, then the Perron-Frobenius eigenvalue $\tau_{\epsilon}$ of $A_{\epsilon}^{m}$ may still be negative for some $\epsilon$. The eigenvalues of $A_{\epsilon}^{m}$ are known to be a convex function of the entries of the diagonal matrix $\epsilon \operatorname{diag}\left(a_{i i}^{m}\right)$. In particular, from Corollary 3 and by the convexity of the max function, it follows that $\tau_{\epsilon} \leq \tau+\epsilon \max _{i} a_{i i}^{m}$. Hence, a lower bound to the minimum allowed (negative) perturbation that maintains box invariance is given by the inequality $\epsilon>-\frac{\tau}{\max _{i} a_{i i}^{m}}$.

2) Off-diagonal Perturbations: In the second case, more complex in general than the first, we can again exploit the upper bounds described in either Thm. 10 or Cor. 3 to make sure the box invariance condition is retained if some of the off diagonal terms vary. Introducing a new perturbed matrix $A_{\epsilon}^{m}$, where $a_{\epsilon, i j}^{m}=a_{i j}^{m}\left(1+\epsilon_{i j}\right), \forall i, j \neq i$ and $a_{\epsilon, i i}^{m}=a_{i i}^{m}$, we are interested in finding how much we can perturb the off diagonal elements of the matrix $A^{m}$, while preserving box invariance. For direction $i$, introducing the vector $\epsilon^{i}=$ $\left[\epsilon_{i j}\right]_{j=1, \ldots, n}$ and a vector $\boldsymbol{v}^{i}=\left[\delta_{i j}\right]_{j=1, \ldots, n}$, where $\delta_{i j}$ is the Kronecker delta, we state the problem as follows:

$$
\begin{array}{ll}
\max _{\boldsymbol{\epsilon}^{i} \geq 0} & \left\|\boldsymbol{\epsilon}^{i}\right\|_{2}^{2} \\
\text { s.t. } & \left.\sum_{j=1}^{n} A_{\boldsymbol{\epsilon}^{i}}^{m}\right|_{(i, j)}<0, \quad \boldsymbol{v}^{i^{T}} \boldsymbol{\epsilon}^{i}=0 .
\end{array}
$$

We arbitrarily chose a particular norm, due to its intuitive meaning. Moreover, we focus on positive perturbations for the off-diagonal terms, because only those can actually affect box invariance. The reader should notice that, while negative perturbations do not affect box invariance, they may interfere with the Metzler structure of the matrix (in particular, its irreducibility). The first constraint above comes from Thm 10. In general, as discussed, it can be substituted by $\left.\left(X^{\tau}\right)^{-1} A_{\epsilon^{i}}^{m} X^{\tau}\right|_{(i, j)} \leq 0, \forall i=1, \ldots, n$, where $X^{\tau}$ is a diagonal matrix formed with the elements of the Perron (right) eigenvector $\boldsymbol{x}^{\tau}$ of $A^{m}$. The second constraint forces the diagonal terms of $A^{m}$ to stay unperturbed, and bound the solution of the problem. The optimization problem can be restated introducing two Lagrange multipliers (respectively $\lambda>\mathbf{0}$ and $\nu$ ), one for each constraint. Let us denote the $i^{\text {th }}$ row of $A_{\boldsymbol{\epsilon}^{i}}^{m}$ as $A_{i}^{m}\left(1+\boldsymbol{\epsilon}^{\boldsymbol{i}}\right)$. Calculations show that the solution has the form, $\boldsymbol{\epsilon}^{i}=\frac{1}{2}\left(\lambda A^{m^{T}}+\gamma \boldsymbol{v}^{i}\right)$, where

$$
\begin{aligned}
& \lambda=\frac{1}{\sum_{j=1, j \neq i}^{n} a_{i j}^{m}}+\frac{a_{i i}^{m}}{\sum_{j=1, j \neq i}^{n}\left(a_{i j}^{m}\right)^{2}} ; \\
& \nu=-\frac{a_{i i}^{m}}{\sum_{j=1, j \neq i}^{n} a_{i j}^{m}}-\frac{\left(a_{i i}^{m}\right)^{2}}{\sum_{j=1, j \neq i}^{n}\left(a_{i j}^{m}\right)^{2}}=-\lambda a_{i i}^{m} .
\end{aligned}
$$


This can be rewritten as follows:

$$
\begin{aligned}
\epsilon_{i}^{i} & =0 ; \\
\epsilon_{j}^{i} & =\frac{1}{2}\left(\frac{a_{i j}^{m}}{\sum_{j=1, j \neq i}^{n} a_{i j}^{m}}+\frac{a_{i i}^{m} a_{i j}^{m}}{\sum_{j=1, j \neq i}^{n}\left(a_{i j}^{m}\right)^{2}}\right) .
\end{aligned}
$$

3) General case: At the cost of not obtaining closed form solutions, we can tackle the problem more generally. Let $A^{m}$ be a Metzler matrix that describes a box invariant linear system. Consider the perturbed matrix $A_{\epsilon}^{m}=A^{m}+E=$ $A^{m}+\sum_{i, j=1}^{n} \epsilon_{i j}\left[\Delta_{(i, j)}\right]$, where $\Delta_{(i, j)}$ is an $n \times n$ matrix that has a 1 in position $(i, j)$, and 0 elsewhere, and $\epsilon_{i j} \geq$ $0, \forall i, j \in 1, \ldots, n$. It is clear that adding positive terms to a Metzler Matrix may disrupt its box invariance; it then makes sense, in order to understand what is the worst (in some sense) perturbation that does not affect the box invariance property, to set up the following problem:

$$
\begin{array}{cl}
\max _{E} & f(E) \\
\text { s.t. } & \left(A_{\epsilon}^{m} \mathbf{1}<\mathbf{0}\right) \vee\left(\mathbf{1}^{T} A_{\epsilon}^{m}<\mathbf{0}\right), \quad E \geq 0 .
\end{array}
$$

Here $f(E)$ is a measure of the "perturbation level"; for instance, we may choose $f(E)=\sum_{i, j=1}^{n} e_{i, j}=\sum_{i, j=1}^{n} \epsilon_{i j}$, or $f(E)=\|E\|_{p}, p \geq 1$. The first constraint codifies the condition of Thm. 10. For the 2-norm $(p=2)$, interpreting $E$ as a function of its elements $\epsilon_{i j}$, introducing an epigraph and resorting to the Schur complement, we can reformulate the problem as the following LMI:

$$
\begin{array}{rl}
\max _{\epsilon_{i j} \geq 0, s \geq 0} & s \\
\text { s.t. } & {\left[\begin{array}{cc}
-s I & -E(\epsilon) \\
E(\epsilon) & s I
\end{array}\right] \succeq 0,} \\
& \min \left\{A_{\epsilon}^{m} \mathbf{1}, \mathbf{1}^{T} A_{\epsilon}^{m}\right\}<\mathbf{0},
\end{array}
$$

where the last inequality ought to be interpreted componentwise.

\section{E. Sensitivity Properties}

Given the Perron eigenvalue $\tau$ of the matrix $A^{m}$ and both left and right Perron eigenvectors $\boldsymbol{x}^{\tau}$ and $\boldsymbol{y}^{\tau}$, we can calculate the sensitivity of $\tau$ with respect to the elements of $A_{\epsilon}^{m}=A^{m}+\epsilon D$. Here $D$ is a fixed matrix of disturbances that are parameterized by $\epsilon$. It can be stated that (cf. [7])

$$
\left.\frac{d \tau}{d \epsilon}\right|_{\epsilon=0}=\frac{\boldsymbol{y}^{\tau^{T}} D \boldsymbol{x}^{\tau}}{\boldsymbol{y}^{\tau^{T}} \boldsymbol{x}^{\tau}} \text { and that } \frac{d \tau}{d\left(a_{\epsilon}^{m}\right)_{i j}}=\frac{\boldsymbol{y}_{i}^{\tau} \boldsymbol{x}_{j}^{\tau}}{\boldsymbol{y}^{\tau^{T}} \boldsymbol{x}^{\tau}} .
$$

\section{F. Affine Systems.}

Consider the affine system, $\dot{\boldsymbol{x}}=A \boldsymbol{x}+\boldsymbol{b}$. We can relate the box invariance of such a system to the condition that the equilibrium point lies in the positive quadrant, $\boldsymbol{x}_{0}>0$. The idea is to exploit the corresponding Metzler matrix $A^{m}$ to deduce possible box properties of the system around $\boldsymbol{x}_{0}$. The assumption of $\boldsymbol{x}_{0}$ being in the positive quadrant is justified both from a technical standpoint and from our applications.

Theorem 11: If the affine system $\dot{\boldsymbol{x}}=A \boldsymbol{x}+\boldsymbol{b}$ has Metzler matrix and $\boldsymbol{b}>\mathbf{0}$, then its equilibrium point $\boldsymbol{x}_{0}>\mathbf{0}$ iff the system is box invariant.
Remark 8: The assumptions of the previous theorem can be relaxed to having a non-negative $\boldsymbol{b} \geq \mathbf{0}, \boldsymbol{b} \neq \mathbf{0}$.

Theorem 12: If the affine system $\dot{\boldsymbol{x}}=A \boldsymbol{x}+\boldsymbol{b}$ has Metzler matrix and its equilibrium point $\boldsymbol{x}_{0}>\mathbf{0}$, then the positivity of its drift term $\boldsymbol{b}>\mathbf{0}$ implies that the system is box invariant. The converse is not true.

The following result is an attempt to get condition for box invariance for an affine system looking at a properly modified correspondent, as we did in the linear case:

Theorem 13: Assume $\boldsymbol{b} \neq 0$. Given an affine dynamical system $\dot{\boldsymbol{x}}=A \boldsymbol{x}+\boldsymbol{b}$, consider the correspondent modified system $\dot{\boldsymbol{x}}=A^{m} \boldsymbol{x}+\boldsymbol{b}^{P}$, where we substituted the Metzler correspondent of $A$ and, additionally, we introduced $\boldsymbol{b}^{P}$, composed of the absolute values of the components of $\boldsymbol{b}$. The original system is box-invariant iff the modified system has a positive equilibrium.

\section{G. Robust Properties of Box Invariance - Affine Systems}

The results on box invariance for affine systems, in particular Thm. 13, show that this property is again intrinsically dependent on the Metzler correspondent of the system matrix. If we consider a perturbed version of the dynamical system $\dot{\boldsymbol{x}}=A \boldsymbol{x}+\boldsymbol{b}$, say $\dot{\boldsymbol{x}}=A_{\epsilon} \boldsymbol{x}+\boldsymbol{b}_{\epsilon}$, its box invariance will be checked on the modified system $\dot{\boldsymbol{x}}=A_{\epsilon}^{m} \boldsymbol{x}+\boldsymbol{b}_{\epsilon}^{P}$. As long as $A_{\epsilon}^{m}$ will remain box invariant the perturbed system will be as well, regardless of the values of vector $\boldsymbol{b}_{\epsilon}^{P}$.

\section{H. Extensions to a class of NonLinear Systems}

In this section we use ideas from the robustness study above to efficiently check box invariance (using only a sufficient, but not necessary, characterization) of a subclass of multi-affine systems in which the degree of each polynomial is at most two. This assumption is natural on models of biochemical reactions in which every reaction can have at most two reactants. We shall tackle the study of these systems leveraging on two different perspectives.

a) NonLinear Systems as perturbations of Linear Systems: Consider a general non linear, multi-affine model $\dot{\boldsymbol{x}}=f(\boldsymbol{x}), \boldsymbol{x} \in \mathbb{R}^{n} ;$ the structure of the vector field allows to express the model as

$$
\dot{\boldsymbol{x}}=A \boldsymbol{x}+g(\boldsymbol{x})=A \boldsymbol{x}+B(\boldsymbol{x}) \boldsymbol{x}=\Gamma(\boldsymbol{x}) \boldsymbol{x} .
$$

where $A$ is a constant $n \times n$ matrix, while $B(\boldsymbol{x})$ is made up of terms that are now linear in the variables, and in particular can be chosen $^{3}$ to have the form $b(\boldsymbol{x})_{i i}=0, b(\boldsymbol{x})_{i j}=$ $\beta x_{i}+\gamma x_{k} ; i \neq k \neq j \in\{1,2, \ldots, n\} ; \beta, \gamma \in \mathbb{R}$. The nullity of the values on the diagonal simply states that a dimerization cannot yield the very same element. Notice that in the offdiagonal positions we could in principle also accomodate second order homogeneous terms $\left(b(\boldsymbol{x})_{i j}=\beta x_{i}+\gamma x_{k}+\delta x_{j}\right.$, which would incidentally disrupt the multi-affine structure as defined in [12]).

Let us now assume that system corresponding to the linear part $(\dot{\boldsymbol{x}}=A \boldsymbol{x})$ is box-invariant, that is there exists a

\footnotetext{
${ }^{3}$ The nonlinear part, which is made up of polinomials of second order, that is products of two different monomials, can be ordered into possibly different $B(\boldsymbol{x})$ matrices. Notice that we avoid introducing indexes on the multiplicative constants $\beta, \gamma$.
} 
nontrivial (conical) set $\mathcal{C}$ in $\mathbb{R}^{n}$ that defines all the possible locations of the symmetric vertices of the invariant hyperrectangle. Let us introduce a matrix $\Gamma^{m}(\boldsymbol{x}) \doteq A^{m}+B^{m}(\boldsymbol{x})$, where $b^{m}(\boldsymbol{x})_{i j}=\left|b(\boldsymbol{x})_{i j}\right|$. It is then possible to refer back to section III-D. 2 and think of $\Gamma^{m}(\boldsymbol{x})=A_{\epsilon}^{m}$, where $b^{m}(\boldsymbol{x})_{i j}=\epsilon_{i j} / a_{i j}^{m}$. In other words, the non-linear part can be conceived as an additional term that may possibly disrupt the box invariance of the linear system. Clearly this is a pessimistic take, which comes from the positivity assumption on the terms $b^{m}(\boldsymbol{x})_{i j}$. By the application of the results in paragraph III-D.2, a set of upper bounds for the values of the allowed "perturbations" is given. Furthermore, these bounds define some hyperplanes which, when intersected, reduce the feasible region for the vertices of the box: $b^{m}(\boldsymbol{x})_{i j}=\mid \beta x_{i}+$ $\gamma x_{k}|\leq| \beta|| x_{i}|+| \gamma|| x_{k} \mid \leq \epsilon_{i j} / a_{i j}^{m}$, where $\epsilon_{i j}$ is here the maximum allowed perturbation, solution of the optimization problem. Notice that these inequalities on halfspaces are all satisfiable on the positive quadrant, and when intersected with the cone $\mathcal{C}$ define a new set of possible vertices for the invariant hyper-rectangle.

Finally, notice that the above procedure can be also extended to include the presence of a constant drift term, analogously to Sec. III-F.

b) Overvaluing Dynamical Systems: A second method to compute invariant regions, closely related to the first in its outcomes, is based on the definition of an overvaluing system [21], [22], which depends on the choice of a particular (vector) norm [5]. Consider the multi-affine model already introduced: $\dot{\boldsymbol{x}}=A \boldsymbol{x}+g(\boldsymbol{x})=A \boldsymbol{x}+B(\boldsymbol{x}) \boldsymbol{x}=\Gamma(\boldsymbol{x}) \boldsymbol{x}, \boldsymbol{x} \in$ $\mathbb{R}^{n}$. As shown in Section II-A, in our study, we are interested in a real valued, infinity vector norm $p(\boldsymbol{x})=\|\boldsymbol{x}\|_{\infty}$ or possibly in a scaled version thereof, $p^{W}(\boldsymbol{x})=\|W \boldsymbol{x}\|_{\infty}$, where $W$ is a diagonal, positive $n \times n$ matrix. The rightderivative of $p(\boldsymbol{x})$ [5], $D^{+} p(\boldsymbol{x})$, can be upper-bounded, within a given limited region $\mathcal{S} \subset \mathbb{R}^{n}$, by a value $m \in \mathbb{R}$ as follows: $D^{+} p(\boldsymbol{x}) \leq m p(\boldsymbol{x})$. Results in [21] allow to claim that, whenever the inequality $D^{+} p(\boldsymbol{x}) \leq m p(\boldsymbol{x})$ holds in $\mathcal{S}$ with $m<0$, then the region defined as

$$
B \doteq\left\{\boldsymbol{x} \in \mathbb{R}^{n}: p(\boldsymbol{x}) \leq c, c \in \mathbb{R}_{+}\right\} \subseteq \mathcal{S}
$$

is positively invariant for the original nonlinear system. As a side result, the original non linear system will be asymptotically stable, as expected. The right-derivative $D^{+} p(\boldsymbol{x})$ can be upper-bounded by a set of inequalities: given the matrices $A$ and $B(\boldsymbol{x})$ as in the preceding paragraph, notice that $D^{+} p(\boldsymbol{x}) \leq \max _{i=1, \ldots, n}\left\{a_{i i}+\sum_{j \neq k \neq i}\left|a_{i j}\right|+|\beta|\left|x_{i}\right|+\right.$ $\left.|\gamma|\left|x_{k}\right|\right\} p(\boldsymbol{x})$. This condition is in fact similar to the one used for the robustness study in section III-D.2 and exploited above; here no prior assumption on the existence of an invariant box is raised. Similarly, accounting for the rescaling factors, for the case of $p^{W}(\boldsymbol{x})$. The above region $B \subseteq \mathcal{S}$ is an $n$-dimensional hypercube with side $2 c$. The vector norm $p^{W}(\boldsymbol{x})$ would instead single out a symmetric hyper rectangle with a vertex lying on the vector $\left[w_{11}, \ldots, w_{n n}\right]^{T}$.

The approach can be similarly extended to the case of a nonlinear vector field with constant drift [21].

\section{Box Invariance and Related Concepts in the Literature.}

We have seen that the concept of box invariance can be looked at from the perspective of (vector) norms inducing non-smooth Lyapunov functions. A more direct relationship is obtained by intersecting proper ellipsoidal functions that have been adequately stretched to the limit, as developed in [3]. We have also already stressed that the concept of box invariance is strongly related to that of componentwise stability [23], [24] and diagonally-invariant stability [25], although here the computational aspects are highlighted, and robustness is originally analyzed. Finally, we mention the connections of the approach here presented with the concepts of practical stability, ultimate boundedness and Lagrange stability. Please refer to [3] for further discussions and pointers to the literature.

\section{Application Instances in Biology}

\section{Example 3: A Model for Blood Glucose Concentration.}

The following model is taken from [26]. It is a model of a physiologic compartment, specifically the human brain, and focuses on the dynamics of the blood glucose concentration. In general, this compartment is part of a network of different parts, which model the concentration in other organs of the body, and which follow some conservation laws that account for the exchange of matter between different compartments. The mass balance equations are the following:

$$
\begin{aligned}
V_{B} \dot{C}_{B o} & =Q_{B}\left(C_{B i}-C_{B o}\right)+P A\left(C_{I}-C_{B o}\right)-r_{R B C} \\
V_{I} \dot{C}_{I} & =P A\left(C_{B o}-C_{I}\right)-r_{T},
\end{aligned}
$$

where $V_{B}$ describes the capillary volume, $V_{I}$ the interstitial fluid volume, $Q_{B}$ the volumetric blood flow rate, $P A$ the permeability-area product, $C_{B i}$ the arterial blood solute concentration, $C_{B o}$ the capillary blood solute concentration, $C_{I}$ the interstitial fluid solute concentration, $r_{R B C}$ the rate of red blood cell uptake of solute, and $r_{T}$ models the tissue cellular removal of solute through cell membrane. The quantity $P A$ can be expressed as the ratio $V_{I} / T$, where $T$ is the transcapillary diffusion time. For this last value, which may in general vary, we choose the value $T=10$ [min].

\begin{tabular}{|l|c|l|c|}
\hline$V_{B}$ & $0.04[\mathrm{l}]$ & $V_{I}$ & $0.45[\mathrm{l}]$ \\
\hline$Q_{B}$ & $0.7[1 / \mathrm{min}]$ & $C_{B i}$ & $0.15[\mathrm{~kg} / \mathrm{l}]$ \\
\hline$r_{T}$ & $2 \times 10^{-6}[\mathrm{~kg} / \mathrm{min}]$ & $r_{R B C}$ & $10^{-5}[\mathrm{~kg} / \mathrm{min}]$ \\
\hline
\end{tabular}

By applications of the conditions described above, the system is box invariant. Figure 2 plots a trajectory and some boxes for this system.

Example 4: The global trafficking model for EGFR and HER2. The following model is taken from [27]. It is affine in its variables, the six dimensional vector $\boldsymbol{x}=$ $\left(R_{s}, C_{s}, H_{s}, R_{i}, C_{i}, H_{i}\right)$, and presents a positive constant drift. The parameter $L$ assumes three possible values, thus we shall just consider its possible different realizations separately. In this particular study, we refer to the instance 

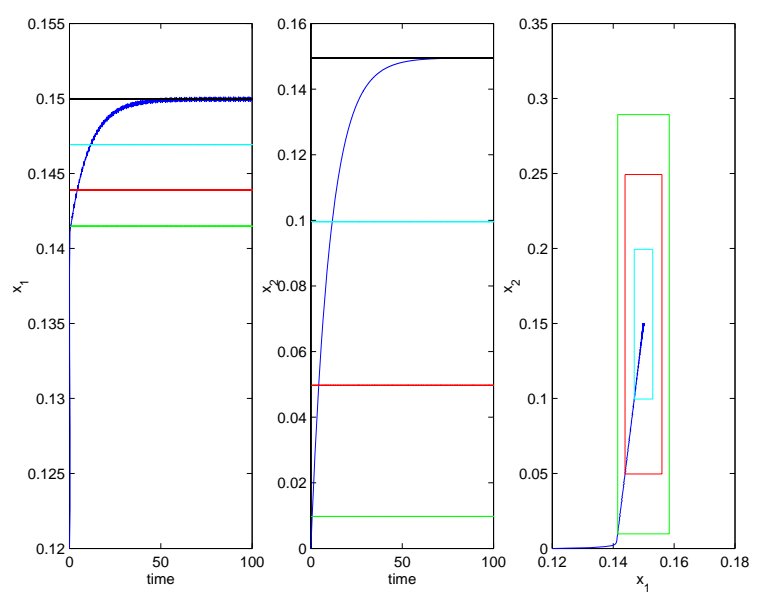

Fig. 2. Blood Glucose Concentration: simulation of a trajectory, and computation of some boxes.

where the quantity of $E G F$ is $L=10^{-12}[\mathrm{ng} / \mathrm{ml}]$.

$$
\begin{aligned}
& d \dot{R}_{s}=S_{R}-k_{f} L R_{s}+k_{e r} C_{s}-k_{e r} R_{s}+k_{x r} f_{x r} R_{i} \\
& d \dot{C}_{s}=k_{f} L R_{s}-k_{r} C_{s}-k_{e c} C_{s}+k_{x c} f_{x c} C_{i} \\
& d \dot{H}_{s}=S_{H}-k_{e h} H_{s}+k_{x h} f_{x h} H_{i} \\
& d \dot{R}_{i}=k_{e r} R_{s}-k_{x r} R_{i} \\
& d \dot{C}_{i}=k_{e c} C_{s}-k_{x c} C_{i} \\
& d \dot{H}_{i}=k_{e h} H_{s}-k_{x h} H_{i}
\end{aligned}
$$

The system matrix verifies the structural conditions on the signs of its elements, as of Thm. 3; given their values, the application of the results above shows the existence of a box. Furthermore, noticing that the system matrix is explicitly Metzler and that the constant drift is positive, we may apply Thm. 11 and conclude that the system is box invariant. A calculation of its eigenvalues shows that they are, as expected, all negative (in particular, the Perron-Frobenius one). Nevertheless, the computation of the Perron-Frobenius eigenvector yields a non positive solution, thus going against the equivalent condition 4) in Prop. 4. Why does that happen? The reason is that the system matrix is not irreducible. In fact, the third and the sixth coordinates are decoupled from all the others; thus, the underlying graph associated with the matrix is not strongly connected. Fortunately, as discussed in Sec. III-A, we can carry on single studies of these two separate components of the system. Introducing $\boldsymbol{x}_{1}=\left(R_{s}, C_{s}, R_{i}, C_{i}\right) \in \mathbb{R}^{4}$ and $\boldsymbol{x}_{2}=\left(H_{s}, H_{i}\right) \in \mathbb{R}^{2}$, we can set up the following reduced models:

$$
\begin{aligned}
\dot{\boldsymbol{x}}_{1} & =\left(\begin{array}{cccc}
-k_{f} L-k_{e r} & k_{r} & k_{x r} f_{x r} & 0 \\
k_{f} L & -k_{r} & 0 & k_{x c} f_{x c} \\
k_{e r} & 0 & -k_{x r} & 0 \\
0 & k_{e c} & 0 & -k_{x c}
\end{array}\right) \boldsymbol{x}_{1}+\left(\begin{array}{c}
S_{R} \\
0 \\
0 \\
0
\end{array}\right) ; \\
\dot{\boldsymbol{x}}_{2} & =\left(\begin{array}{cc}
-k_{e h} & k_{x h} f_{x h} \\
k_{e h} & -k_{x h}
\end{array}\right) \boldsymbol{x}_{2}+\left(\begin{array}{c}
S_{H} \\
0
\end{array}\right) .
\end{aligned}
$$

The two new reduced-size system matrices are irreducible, Metzler, and verify all the equivalent conditions for box invariance of Prop. 4. This example should advise the reader about the applicability of the conditions derived for Metzler matrices. The instances where they do not completely hold suggest ways to structurally simplify the analysis of the problem.

\section{REFERENCES}

[1] F. Blanchini, "Set invariance in control," Automatica, vol. 35, pp. 1747-1767, 1999.

[2] A. Podelski and S. Wagner, "Model checking of hybrid systems: from reachabilty towards stabilty," in Hybrid Systems: Computation and Control, ser. LNCS 3927. Springer, Apr 2006.

[3] A. Abate, A. Tiwari, and S. Sastry, "The concept of box invariance for biologically-inspired dynamical systems," EECS Dept., UC Berkeley, Tech. Rep. UCB/EECS-2006-185, 2006. [Online]. Available: http://www.eecs.berkeley.edu/Pubs/TechRpts/2006/EECS2006-185.html

[4] O. Pastravanu and M. Voicu, "Norm-based approach to componentwise asymptotic stability," in Proc. 11th IEEE Mediterranean Conf. on Control and Automation, 2003.

[5] H. Kiendl, J. Adamy, and P. Stelzner, "Vector norms as lyapunov functions for linear systems," IEEE Transactions on Automatic Control, vol. 37, pp. 839-842, 1992.

[6] K. Loskot, A. Polanski, and R. Rudnicki, "Further comments on "vector norms as Lyapunov functions for linear systems"," IEEE Trans. on Automatic Control, vol. 43, no. 2, 1998.

[7] R. Horn and C. Johnson, Matrix Analysis. Cambridge University Press, 1985.

[8] G. Coxson, "The p-matrix problem is co-np-complete," Mathematical Programming, vol. 64, pp. 173-178, 1994.

[9] - "Computing exact bounds on elements of an inverse interval matrix is np-hard," Reliable Computing, vol. 5, pp. 137-142, 1999.

[10] A. Tarski, A Decision Method for Elementary Algebra and Geometry. University of California Press, 1948, second edition.

[11] G. E. Collins, "Quantifier elimination for the elementary theory of real closed fields by cylindrical algebraic decomposition," in Proc. 2nd GI Conf. Automata Theory and Formal Languages, ser. LNCS, vol. 33. Springer, 1975, pp. 134-183.

[12] C. Belta, L. Habets, and V. Kumar, "Control of multi-affine systems on rectangles with applications to hybrid biomolecular networks," in Proc. 41st Conf. on Decision and Control. IEEE, 2002, pp. 534-539.

[13] P. Lincoln and A. Tiwari, "Symbolic systems biology: Hybrid modeling and analysis of biological networks," in Hybrid Systems: Computation and Control, ser. LNCS 2993. Springer, 2004, pp. 660-672.

[14] O. Bernard and J.-L. Gouze, "Global qualitative description of a class of nonlinear dynamical systems," Artificial Intelligence, vol. 136, pp. 29-59, 2002.

[15] A. Abate and A. Tiwari, "Box invariance for hybrid and switched systems," in Proceedings of the 2nd IFAC Conference on Analysis and Design of Hybrid Systems, 2006.

[16] A. Julius, A. Halasz, V. Kumar, and G. Pappas, "Controlling biological systems: the lactose regulation system of Escherichia Coli," in American Control Conference 2007, 2007.

[17] O. Perron, "Zur theorie der matrizen," Math. Ann., vol. 64, pp. 248263, 1907.

[18] G. Frobenius, "Über matrizen aus positiven elementen," S.B. Preuss Akad. Wiss. Berlin, pp. 471-476, 1908.

[19] E. Seneta, Nonnegative Matrices. Wiley, New York, 1973.

[20] H. Minc, Nonnegative Matrices. Wiley, New York, 1988.

[21] P. Borne, J. Richard, and N. Radhy, "Stability, stabilization, regulation using vector norms," J. Fossard and D. Normand-Cyrot eds., Nonlinear Systems, Stability and Stabilization, vol. 2, pp. 45-90, 1996.

[22] E. Erdem and A. Alleyne, "Estimation of stability regions of sdre controlled systems using vector norms," in Proceedings of the American Control Conference, 2002, pp. 80-85.

[23] M. Voicu, "Componentwise asymptotic stability of linear constant dynamical systems," IEEE Transactions on Automatic Control, vol. 29, pp. $937-939,1984$.

[24] O. Pastravanu and M. Voicu, "Necessary and sufficient conditions for componentwise stability of interval matrix systems," IEEE Transactions on Automatic Control, vol. 49, pp. 1016-1021, 2004.

[25] O. Pastravanu, M. Matcovschi, and M. Voicu, "Diagonally-invariant exponential stability," in Proceedings of the 16th IFAC World Congress, 2005.

[26] J. Sorensen, "A physiologic model of glucose metabolism in man and its use to design and assess improved insulin therapies for diabetes," Ph.D. dissertation, MIT, 1985.

[27] B. Hendriks, G. Orr, A. Wells, H. Wiley, and D. Lauffenburger, "Parsing erk activation reveals quantitatively equivalent contributions from epidermal growth factor receptor and her2 in human mammary epithelial cells," Biological Chemistry, vol. 280, 7, pp. 6167-6169, 2005. 